\title{
On the multiplication operators on spaces of analytic functions
}

\author{
by \\ B. Yousefi and S. Foroutan (Shiraz) \\ Dedicated to the memory of Professor Karim Seddighi
}

\begin{abstract}
We consider Hilbert spaces of analytic functions on a plane domain $\Omega$ and multiplication operators on such spaces induced by functions from $H^{\infty}(\Omega)$. Recently, $\mathrm{K}$. Zhu has given conditions under which the adjoints of multiplication operators on Hilbert spaces of analytic functions belong to the Cowen-Douglas classes. In this paper, we provide some sufficient conditions which give the converse of the main result obtained by K. Zhu. We also characterize the commutant of certain multiplication operators.
\end{abstract}

Introduction. Let $H$ be a Hilbert space of functions analytic on a plane domain $\Omega$ such that:

(1) Every point $w \in \Omega$ is a nonzero bounded linear functional on $H$, so that $H$ has a reproducing kernel $k_{w} \in H$ such that $f(w)=\left\langle f, k_{w}\right\rangle$ for all $f \in H$.

(2) If $f$ is a function in $H$, then so is $z f$.

(3) If $f \in H$ and $f(\lambda)=0$, then there is a function $g \in H$ such that $(z-\lambda) g=f$.

A space $H$ satisfying the above conditions is called a Hilbert space of analytic functions on $\Omega$. The Hardy and Bergman spaces are examples of Hilbert spaces of analytic functions on the open unit disk.

A complex-valued function $\phi$ on $\Omega$ for which $\phi f \in H$ for every $f \in H$ is called a multiplier of $H$, and every multiplier $\phi$ on $H$ determines a multiplication operator $M_{\phi}$ on $H$ by $M_{\phi} f=\phi f, f \in H$. The set of all multipliers of $H$ is denoted by $M(H)$. Clearly $M(H) \subset H^{\infty}(\Omega)$, where $H^{\infty}(\Omega)$ is the space of all bounded analytic functions on $\Omega$. In fact $\|\phi\|_{\infty} \leq\left\|M_{\phi}\right\|$. A good source on this topic is [4].

2000 Mathematics Subject Classification: 47B38, 47A10.

Key words and phrases: Hilbert space of analytic functions, essential spectrum, Fredholm operator, Cowen-Douglas class of operators, commutant. 
Recall that for a positive integer $n$ and a domain $U \subset \mathbb{C}$, the CowenDouglas class $B_{n}(U)$ consists of bounded linear operators $T$ on any fixed separable infinite-dimensional Hilbert space $X$ with the following properties:

(a) $\operatorname{ran}(\lambda-T)=X$ for every $\lambda \in U$.

(b) $\operatorname{dim}(\operatorname{ker}(\lambda-T))=n$ for every $\lambda \in U$.

(c) $\operatorname{span}\{\operatorname{ker}(\lambda-T): \lambda \in U\}=X$.

Here span denotes the closed linear span of a collection of sets in $X$. For the study of the Cowen-Douglas classes $B_{n}$ we mention [1], [2] and [5].

Also recall that a bounded linear operator $\mathrm{A}$ on a Hilbert space is a Fredholm operator if and only if $\operatorname{ran} A$ is closed and both $\operatorname{ker} A$ and $\operatorname{ker} A^{*}$ are finite-dimensional. We use $\sigma(A)$ and $\sigma_{\mathrm{e}}(A)$ to denote respectively the spectrum and the essential spectrum of $A$.

Main results. In Propositions 7 and 10 of [6], K. Zhu gives sufficient conditions for the adjoint of a multiplication operator on Hilbert spaces of analytic functions to belong to the Cowen-Douglas class $B_{n}$ for a positive integer $n$. In this paper we investigate the converse of Zhu's results. Also we consider the commutant of special multiplication operators. First, for the benefit of the reader we give the main result of [6]:

TheOREm 1. Suppose $\phi \in H^{\infty}(\Omega)$ and $V$ is a domain contained in $\phi(\Omega)$. If there exists a positive integer $n$ such that $\Omega \cap \phi^{-1}(\lambda)$ consists of $n$ points (counting multiplicity) for every $\lambda \in V$, then the adjoint of the operator $M_{\phi}: H \rightarrow H$ belongs to the Cowen-Douglas class $B_{n}(U)$, where $U=\{\bar{z}: z \in V\}$.

Proof. See Proposition 10 in [6].

In the rest of the paper we assume that $H$ is a Hilbert space of analytic functions on a bounded plane domain $\Omega$. We further assume that

$$
M(H)=H^{\infty}(\Omega) .
$$

In the following by $K_{\lambda}$ we mean the unit vector $k_{\lambda} /\left\|k_{\lambda}\right\|$.

Theorem 2. Suppose $\phi \in H^{\infty}(\Omega)$ and $V$ is a domain in $\mathbb{C}$ such that, for a positive integer $n$, the adjoint of the operator $M_{\phi}: H \rightarrow H$ belongs to the Cowen-Douglas class $B_{n}(U)$, where $U=\{\bar{z}: z \in V\}$. Also suppose that the convergence of any sequence $\left\{z_{n}\right\}_{n} \subset \Omega$ to a boundary point of $\Omega$ implies the weak convergence of $\left\{K_{z_{n}}\right\}_{n}$. Then $V \subset \phi(\Omega)$ and $\Omega \cap \phi^{-1}(\lambda)$ consists of $n$ points (counting multiplicity) for every $\lambda \in V$.

Proof. First note that closure $(\phi(\Omega)) \subset \sigma\left(M_{\phi}\right)$. Also if $\lambda \in \phi(\Omega)$, then $\lambda=\phi\left(\lambda_{0}\right)$ for some $\lambda_{0} \in \Omega$ and so $M_{\phi}^{*} k_{\lambda_{0}}=\bar{\lambda} k_{\lambda_{0}}$. Thus $\phi(\Omega) \subset \sigma\left(M_{\phi}\right)$ and indeed $\sigma\left(M_{\phi}\right)=\operatorname{closure}(\phi(\Omega))$ and $M_{\phi}^{*}-\lambda$ is Fredholm for all $\lambda \in U$. Thus we get $V \subset$ closure $(\phi(\Omega)) \backslash \sigma_{\mathrm{e}}\left(M_{\phi}\right)$. Now to prove that $V \subset \phi(\Omega)$ it 
is sufficient to show that $\partial \phi(\Omega) \subset \sigma_{\mathrm{e}}\left(M_{\phi}\right)$. For this let $\lambda \notin \sigma_{\mathrm{e}}\left(M_{\phi}\right)$ and also let $\left\{z_{n}\right\}_{n}$ be a sequence in $\Omega$ such that $\left\{z_{n}\right\}_{n}$ converges to a point in $\partial \Omega$ (boundary of $\Omega$ ) and $\phi\left(z_{n}\right) \rightarrow \lambda$. Then by the relation

$$
M_{\phi-\lambda}^{*} K_{z_{n}}=\left(\overline{\phi\left(z_{n}\right)}-\bar{\lambda}\right) K_{z_{n}}
$$

we get $\left\|\left(M_{\phi}^{*}-\bar{\lambda}\right) K_{z_{n}}\right\| \rightarrow 0$, which is a contradiction, because $M_{\phi}^{*}-\bar{\lambda}$ is Fredholm and the sequence of unit vectors $\left\{K_{z_{n}}\right\}_{n}$ tends weakly to zero. This implies that $\partial \phi(\Omega) \subset \sigma_{\mathrm{e}}\left(M_{\phi}\right)$ and so $V \subset \phi(\Omega)$.

Now we prove the second part of the theorem. If $\lambda \in V$, then $\lambda=\phi\left(\lambda_{0}\right)$ for some $\lambda_{0} \in \Omega$ and so $M_{\phi}^{*} k_{\lambda_{0}}=\bar{\lambda} k_{\lambda_{0}}$. Thus $k_{\lambda_{0}} \in \operatorname{ker}\left(M_{\phi}-\lambda\right)^{*}$. Since $\operatorname{dim}\left(\operatorname{ker}\left(M_{\phi}-\lambda\right)^{*}\right)=n$ and a finite subset of points $w$ in $\Omega$ yields a linearly independent set of functions $k_{w}$ in $H$, the set $\Omega \cap \phi^{-1}(\lambda)$ consists of at most $n$ points for all $\lambda \in V$. So for each fixed $\lambda \in V$, there exist $\lambda_{1}, \ldots, \lambda_{m}$ in $\Omega$ and $n_{1}, \ldots, n_{m}$ in $\mathbb{N}$ such that $m \leq n$ and for all $z \in \Omega$ we have

$$
\phi(z)-\lambda=\psi(z)\left(z-\lambda_{1}\right)^{n_{1}}\left(z-\lambda_{2}\right)^{n_{2}} \cdots\left(z-\lambda_{m}\right)^{n_{m}},
$$

where $\psi$ belongs to $H^{\infty}(\Omega)$ and is nonvanishing on $\Omega$. The function $\psi$ is also bounded below on $\Omega$. In fact, if we choose $r>0$ such that the closed disk $D$ centered at $\lambda$ with radius $r$ is contained in $V$, then the pre-image in $\Omega$ of $D$ under $\phi$ is a compact set $C$ in $\Omega$, which must have a positive distance $\delta$ to $\partial \Omega$. Now if $\psi$ is not bounded below on $\Omega$, then there exists a sequence $\left\{z_{n}\right\}$ in $\Omega \backslash\left\{\lambda_{1}, \ldots, \lambda_{m}\right\}$ such that $\psi\left(z_{n}\right) \rightarrow 0$ as $n \rightarrow \infty$. Since $\psi$ is nonvanishing on $\Omega$, we must have $z_{n} \rightarrow \partial \Omega$ as $n \rightarrow \infty$. On the other hand, the boundedness of $\Omega$ implies that $\phi\left(z_{n}\right) \rightarrow \lambda$, so there exists a positive integer $N$ such that $\phi\left(z_{n}\right) \in D$ for all $n>N$, or $z_{n} \in C$ for all $n>N$, a contradiction to $z_{n} \rightarrow \partial \Omega$. Since $\psi$ is bounded above and below on $\Omega$, the operator $M_{\psi}$ is invertible on $H$ and so

$$
\operatorname{index}\left(M_{\psi}\right)=\operatorname{dim}\left(\operatorname{ker} M_{\psi}\right)-\operatorname{dim}\left(\operatorname{ker} M_{\psi}^{*}\right)=0 .
$$

Now by the relation $(*)$ we have

$$
\operatorname{index}\left(M_{\phi-\lambda}^{*}\right)=\sum_{j=1}^{m} n_{j}\left(\operatorname{index}\left(M_{z-\lambda_{j}}^{*}\right)\right) .
$$

Since $M_{\phi}^{*} \in B_{n}(U)$ and $\lambda \in V$ we get

$$
\operatorname{index}\left(M_{\phi-\lambda}^{*}\right)=-\operatorname{dim}\left(\operatorname{ker} M_{\phi-\lambda}^{*}\right)=-n .
$$

Also we have

$$
\operatorname{index}\left(M_{z-\lambda_{j}}^{*}\right)=-\operatorname{dim}\left(\operatorname{ker} M_{z-\lambda_{j}}^{*}\right)
$$

for $j=1, \ldots, m$ and by condition $(3)$ on $H, \operatorname{ker}\left(M_{z}-\lambda_{j}\right)^{*}$ is one-dimensional ([3]), thus $\sum_{j=1}^{m} n_{j}=n$ and so $\Omega \cap \phi^{-1}(\lambda)$ consists of exactly $n$ points (counting multiplicity) for every $\lambda \in V$. This completes the proof. 
In the following let $\Omega$ be such that if $\lambda \in \Omega$ then $-\lambda \in \Omega$. Also we assume that the composition operator $C_{-z}: H \rightarrow H$ defined by $C_{-z} f=f(-z)$ is bounded.

Proposition 3. Suppose that $\phi \in H^{\infty}(\Omega)$ and there exists a domain $V \subset \phi(\Omega)$ such that $\Omega \cap \phi^{-1}(w)$ is a singleton for every $w \in V$. If $\phi$ is odd and $S M_{\phi}=-M_{\phi} S$, then $S=M_{h} C_{-z}$ for some $h \in H^{\infty}(\Omega)$.

Proof. For any $w \in V$ there exists a unique $\lambda \in \Omega$ such that $w=\phi(\lambda)$. Thus $\phi(z)+w=(z+\lambda) \psi(z)$, where $\psi$ is an invertible function in $H^{\infty}(\Omega)$, and so

$$
\operatorname{dim}\left(\operatorname{ker} M_{\phi+\phi(\lambda)}^{*}\right)=\operatorname{dim}\left(\operatorname{ker} M_{z+\lambda}^{*}\right)=1 .
$$

Since $S M_{\phi}=-M_{\phi} S$, we can see that $M_{\phi}^{*} S^{*} k_{\lambda}=-\overline{\phi(\lambda)} S^{*} k_{\lambda}$ and so $S^{*} k_{\lambda} \in$ $\operatorname{ker} M_{\phi+\phi(\lambda)}^{*}$. But $\operatorname{ker} M_{\phi+\phi(\lambda)}^{*}$ is equal to the closed linear span of $\left\{k_{-\lambda}\right\}$, thus there exists a function $h$ defined on $U \cap \phi^{-1}(V)$ such that $S^{*} k_{\lambda}=$ $\overline{h(\lambda)} k_{-\lambda}$. By the same method used in the proof of Proposition 8 in [6], $h$ can be extended to an analytic function (still denoted $h$ ) on $\Omega$. Therefore we have

$$
\begin{aligned}
S f(\lambda)=\left\langle S f, k_{\lambda}\right\rangle & =\left\langle f, S^{*} k_{\lambda}\right\rangle=\left\langle f, \overline{h(\lambda)} k_{-\lambda}\right\rangle \\
& =h(\lambda) f(-\lambda)=\left(M_{h} C_{-z} f\right)(\lambda) .
\end{aligned}
$$

Thus $S=M_{h} C_{-z}$ and indeed $h \in H^{\infty}(\Omega)$. This completes the proof.

THEOREM 4. Let $\phi \in H^{\infty}(\Omega)$ be an odd map and suppose that for a domain $V \subset \phi(\Omega)$ the set $\Omega \cap \phi^{-1}(w)$ is a singleton for every $w \in V$. If $S M_{\phi^{2}}=M_{\phi^{2}} S$ and $S M_{\phi}-M_{\phi} S$ is compact, then $S=M_{h}$ for some $h \in H^{\infty}(\Omega)$.

Proof. Clearly we can see that $T M_{\phi}=-M_{\phi} T$, where $T=S M_{\phi}-M_{\phi} S$. So by Proposition 3 , there exists $\psi \in H^{\infty}(\Omega)$ such that $T=M_{\psi} C_{-z}$. Since $T$ is compact, $M_{\psi}$ is also compact and by the Fredholm alternative, $\psi=0$. Thus $S M_{\phi}=M_{\phi} S$ and by Proposition 8 in [6], $S=M_{h}$ for some $h \in H^{\infty}(\Omega)$ and so the proof is complete.

Acknowledgements. The authors are grateful to the referee for helpful suggestions.

\section{References}

[1] M. Cowen and E. Douglas, Complex geometry and operator theory, Acta Math. 141 (1978), 187-261.

[2] P. Curto and N. Salinas, Generalized Bergman kernels and the Cowen-Douglas theory, Amer. J. Math. 106 (1984), 447-488.

[3] S. Richter, Invariant subspaces in Banach spaces of analytic functions, Trans. Amer. Math. Soc. 304 (1987), 585-616. 
[4] A. Shields and L. Wallen, The commutants of certain Hilbert space operators, Indiana Univ. Math. J. 20 (1971), 777-788.

[5] K. Zhu, Operators in Cowen-Douglas classes, Illinois J. Math. 44 (2000), 767-783.

[6] - Irreducible multiplication operators on spaces of analytic functions, J. Operator Theory 51 (2004), 377-385.

Department of Mathematics

College of Sciences

Shiraz University

Shiraz 71454, Iran

E-mail: byousefi@shirazu.ac.ir

foroutan@math.susc.ac.ir

Received August 19, 2004

Revised version December 22, 2004 